\title{
Enforcing QoS: Myth or Reality?
}

\author{
Organizers: Gabi Dreo Rodosek ${ }^{1}$ and Metin Feridun ${ }^{2}$ \\ 1 Munich Network Management Team \\ Leibniz Supercomputing Center \\ Barer Str. 21, 80333 Munich, Germany \\ dreo@lrz.de \\ 2 IBM Research \\ Zurich Research Laboratory \\ Saumerstr. 4, 8803 Rueschlikon, Switzerland \\ fer@zurich.ibm.com
}

In recent years, the research community has investigated how quality of service (QoS) can be integrated into services such that it can be managed, i.e., configured, monitored and enforced. The gradual growth in network-based services is making it necessary to implement some notion of quality of service into products such that service providers can differentiate their services, for example, provide different classes of service, where a service is end-to-end. Are research results, approaches sufficient to address this need? Do we know how to provide customer-oriented quality of service? Do we know what quality of service parameters are demanded by customers, which of them provide useful and relevant information? How can we specify and measure these QoS parameters? What are the appropriate approaches? 